\title{
Saccharine preference in rats as a function of age and early experience
}

FRANCIS B..COLAVITA, University of Pittsburgh, Pittsburgh, Pa. 15213

Preference for a $0.13 \%$ saccharine solution over tap water in five groups of rats was seen to vary as a function of age at the start of preference testing, and age when removed from the mother. Previous experience with saccharine had little or no effect on saccharine preference. Rats given 165 successive days of exposure to saccharine showed no tendency for saccharine preference to decline over time. The results suggest that saccharine preference in rats occurs because the sweet taste of saccharine is a primary reward; however, preference magnitude appears to depend upon maturational factors.

It is a well documented fact that adult rats given access to both water and moderate concentrations of saccharine solution will consume more of the saccharine. The underlying basis for this preference for a non-nutritive substance is not understood at the present time. Sheffield \& Roby (1950) have proposed that sweet taste is a primary reward, while Smith \& Capretta (1956) have suggested that saccharine preference is a secondary effect that can be manipulated by altering the animal's maintenance schedule.

The present experiment attempts to further our understanding of saccharine preference in rats by investigating some variables not considered in earlier studies. These variables include: (a) age of animals at initiation of preference testing, (b) previous experience with saccharine, (c) number of days over which testing is carried out, and (d) age of animals when separated from the mother.

Variables (a) and (b) were chosen to determine whether or not maturation plays a role in saccharine preference. Variable (c) was included because of the possibility that long term experience with saccharine would have some effect on its preference over water; finally, variable (d) was studied because of an interesting effect observed during a pilot study when a mother rat died before her pups had reached the usual age for weaning.

\section{Subjects}

\section{METHOD}

The Ss were 41 Sprague-Dawley albino rats from five different litters, all born in the animal colony at the University of Pittsburgh. For four days prior to giving birth, the five pregnant mother rats were housed in individual cages, allowed unlimited access to food (Wayne's Lab Blox), and provided with either a $0.13 \%$ saccharine solution $(1.30 \mathrm{~g}$ of sodium saccharine powder in 11 of tap water) or plain tap water, depending upon what treatment their pups were scheduled to receive. The newborn pups were allowed to remain with the mother rats for 30 days (with the exception of eight animals randomly picked from the five litters who were removed after 20 days), and then placed in individual cages.

\section{Procedure}

The 41 animals were divided into five different treatment groups, designated as follows: Group $30 \mathrm{~S}$, Group $30 \mathrm{~W}$, Group 95S, Group 95W, and Group E. Group 30S was composed of 10 animals that had drunk only saccharine solution for the first 30 days of life. On the 31 st day, both saccharine and water were made available and preference testing was begun. Group 30W contained seven animals raised on water for the first 30 days of life and tested similarly on Day 31 . Group $95 \mathrm{~S}$ consisted of eight animals who had drunk only the saccharine solution for the first 95 days of life before testing was initiated on Day 96. Group 95W was the comparable group raised on water that had not tasted saccharine until the first day of preference testing. Finally, Group $E$ (for early removal) contained the eight animals removed from their mothers at 20 days of age. These rats were kept in a communal cage for an additional 10 days, with access to both saccharine and water. Then, they were moved to individual cages and preference testing was carried out. This group was included because in a pilot study it was noted that rat pups who had lost their mother at 20 days of age drank more saccharine at $\mathbf{3 0}$ days of age than did pups removed from their mother at 30 days of age.

Preference testing was carried out in the same manner for all animals. They were housed in individual cages equipped with two $70 \mathrm{ml}$ drinking tubes, one tube containing tap water and the other containing $0.13 \%$ saccharine solution. The rats were permitted unlimited access to food. Once every $24 \mathrm{~h}$ the amount of saccharine and water imbibed by each animal was recorded. At this time the drinking tubes were emptied, refilled with fresh saccharine or water, and had their positions on the front of the cage changed in accordance with a predetermined schedule to control for left-right position preferences. The saccharine solution was always made up one day ahead of time and allowed to sit overnight. The water for the following day was also allowed to sit overnight so that there were no differences in freshness or temperature between the two test solutions.

Preference testing was carried out for $\mathbf{4 0}$ successive days for all groups. Testing was conducted for an additional $\mathbf{3 0}$ days for Group $95 \mathrm{~S}$ to study the effects of long term exposure on saccharine preference.

\section{RESULTS}

The total volume of liquid consumed by the 95 day old rats was considerably greater than that consumed by the 30 day old rats. For comparison purposes, the data reported below will be based on proportions rather than volume. That is, the formula volume of saccharine drunk/volume of saccharine + water drunk was used to obtain the preference values presented in Table 1 . Also included in this table are the data pertaining to position preference, calculated by taking the absolute value of (volume of saccharine + water drunk on right $-.500 /$ total volume of saccharine + water).

An analysis of variance of the position preference data indicated that none of the five groups of animals demonstrated position preferences greater than could be expected by chance. Some between-group differences in saccharine preference were discovered however. While all groups showed some preference for the saccharine, an analysis of variance showed that significant differences existed in magnitude of saccharine preference $(F=6.55$, df $=4 / 36, p<.01)$. Multiple $t$ comparisons were made among the means of the various treatment groups to study the effects of age at time of testing, prior experience with saccharine, and early removal from the mother, as related to saccharine preference. Table 2 shows the different comparisons made. A two-tailed test was used to evaluate these data. The data presented in Table 2 show that age of the animal at the time of testing, and age at time of removal from the mother, are both critical variables in determining the magnitude of the preference for saccharine. Prior experience with saccharine (the $S$ vs $W$ comparisons) had no apparent effect on later preference for saccharine.

The results of the additional 30 days of preference testing given to the eight animals in Group $95 \mathrm{~S}$ are shown in Table 3. The proportion data showing the magnitude of saccharine preference are presented in 10 day blocks to allow a sequential comparison to be made over the total period of testing, which was 70 days. The block means presented in Table 3 show no tendency for saccharine

Table 1

Mean Proportion Saccharine Preference and Position Preference for All Groups

\begin{tabular}{lcc} 
Group & $\begin{array}{c}\text { Proportion } \\
\text { Saccharine Preference }\end{array}$ & $\begin{array}{c}\text { Proportion } \\
\text { Position Preference }\end{array}$ \\
\hline $30 S$ & .719 & .564 \\
$30 \mathrm{~W}$ & .737 & .515 \\
$95 \mathrm{~S}$ & .910 & .538 \\
$95 \mathrm{~W}$ & .844 & .516 \\
$\mathrm{E}$ & .920 & .512 \\
\hline
\end{tabular}


Table 2

Multiple Comparisons Among Means of Treatment Groups

\begin{tabular}{lc} 
Comparison & $t$ \\
30S \& $30 \mathrm{~W}$ vs $95 \mathrm{~S} \& 95 \mathrm{~W}$ & $.09^{*}$ \\
$30 \mathrm{~S}$ vs $30 \mathrm{~W}$ & 1.76 \\
$95 \mathrm{~S}$ vs $95 \mathrm{~W}$ & $5.13^{*}$ \\
\hline E vs $305 \& 30 \mathrm{~W}$ &
\end{tabular}

$*)<. \|$

preference to change in magnitude as a function of the length of time over which testing is carried out.

Finally, a comparison was made of the mean proportion saccharine preference over Days 1-10 and 31-40 of testing for Groups $30 \mathrm{~S}$ and $30 \mathrm{~W}$. Since these two groups showed an overall saccharine preference significantly below the other three groups, it was felt that this comparison might disclose any tendency for saccharine preference to increase as a function of age, thereby serving as an additional indicator of any role played by maturation in the magnitude of saccharine preference. For Group $30 \mathrm{~S}$ the proportion magnitudes for the first and last 10 days of testing were .616 and .776 , respectively. The corresponding values for Group $30 \mathrm{~W}$ were .640 and .833 . These data indicate that there was an increase in saccharine preference from the first 10 test days to the last 10 test days. As this was a post hoc comparison, no statistical test for significance was used; however, the data are clearly compatible with the finding noted above that 95 day old rats have a greater preference for saccharine than do 30 day old rats.

\section{DISCUSSION}

While it is evident that rats generally prefer a $0.13 \%$ saccharine solution to tap water when given free access to both, the present

Table 3

Proportion Saccharine Preference for Individual Subjects in Group 95S

\begin{tabular}{ccccccccc} 
& \multicolumn{8}{c}{ 10 Day Blocks } \\
\cline { 2 - 9 } Subject & 1 & 2 & 3 & 4 & 5 & 6 & 7 & Mean \\
\hline A & .887 & .883 & .884 & .881 & .913 & .863 & .937 & .891 \\
B & .916 & .896 & .949 & .938 & .911 & .879 & .901 & .912 \\
C & .920 & .934 & .878 & .899 & .905 & .886 & .896 & .902 \\
D & .873 & .873 & .881 & .882 & .827 & .918 & .887 & .877 \\
E & .840 & .860 & .908 & .963 & .908 & .937 & .873 & .898 \\
F & .905 & .943 & .926 & .976 & .925 & .911 & .945 & .933 \\
G & .921 & .947 & .950 & .934 & .951 & .958 & .950 & .944 \\
H & .931 & .892 & .898 & .912 & .934 & .954 & .961 & .916 \\
\hline Mean & .899 & .904 & .909 & .924 & .921 & .913 & .918 & .911 \\
\hline
\end{tabular}

data clearly show that the animal's age is a significant variable in determining the magnitude of this preference. Rats tested at 95 days of age showed higher preference scores than did 30 day rats regardless of whether or not they had had previous experience with saccharine. It might be noted in passing that had a one-tailed test been used rather than a two-tailed test, the mean difference in saccharine preference between Groups $95 \mathrm{~S}$ and $95 \mathrm{~W}$ would have been significant at the .05 level. This suggests that perhaps previous experience with saccharine may also have some in fluence on the magnitude of saccharine preference. The observations made on Groups $30 \mathrm{~S}$ and $30 \mathrm{~W}$ showing greater saccharine preference at 60 days than at 30 days also suggest the importance of age as a factor in magnitude of saccharine preference.

An interesting finding of the present study was the fact that removing a baby rat from its mother at 20 days of agc instead of at 30 days of age seemed to result in an increase in its preference for saccharine. By way of speculation such a finding may indicate that exposure to the mother rat's milk much beyond 20 days results in a temporary alteration in the baby rat's receptivity to sweet substances that is not found in rats weaned at 20 days. Such a mechanism, if it exists would be operative at just the right time to facilitate weaning, which normally occurs some time between the third and fourth week (Rheingold, 1963).

Finally, as is shown in Table 3, extending to 70 days the period of time over which saccharine preference testing is carried out has no apparent effect on the degree of preference shown by rats started at 95 days of age. It will be recalled that since Group $95 \mathrm{~S}$ had had only saccharine available to drink for the first 95 days of life, their total period of exposure to saccharine was 165 successive days. The lack of diminution in saccharine preference after so long a time suggests that in line with the belief of Sheffield \& Roby (1950), sweet taste is indeed a primary reward in rats, and that secondary factors if present play a lesser role. In the cases of Groups $30 \mathrm{~W}$ and $95 \mathrm{~W}$, for instance, water had a long history of being associated with hunger and thirst reduction, while saccharine initially had no such association; yet both groups showed a preference for the saccharine solution. If taste preference were based on previous association with primary drive reduction, one would expect that the above two groups should initially prefer water to saccharine.

\section{REFERENCES}

RHEINGOLD, H. L. (Ed.), Maternal behavior in mammals. New York: Wiley, 1963.

SHEFFIELD, F. D., \& ROBY, T. Reward value of a non-nutritive sweet taste. Journal of Comparative \& Physiological Psychology, 1950, 43, 471-481.

SMITH, M. P., \& CAPRETTA. P. J. Effects of drive level and experience on the reward value of saccharine solutions. Journal of Comparative \& Physiological Psychology, 1956, 49, 553-557. 